\title{
Rapid mobilisation following total hip and knee arthroplasty
}

\author{
B Riemer MBChB(UCT), FC Orth(SA), FRCS Tr\&Orth(Eng) \\ Arthroplasty fellow \\ K MacIntyre MBChB(US), FC Orth(SA) \\ Arthroplasty fellow \\ M Nortje MBChB(UCT), FC Orth(SA), MMed(UCT) \\ Consultant surgeon \\ B Dower MBChB(UCT), FCS Orth(SA) \\ Consultant surgeon \\ G Grobler MBChB(UCT), FRCS(Edin), FCS Orth(SA), MMed(UCT) \\ Consultant surgeon
}

M Springfield BSc(Physio)Wits, MBChB(Wits), FCA(SA), MSc(Health informatics)UCL

Consultant anaesthetist

Division of Orthopaedic Surgery, Groote Schuur Hospital, Cape Town

\author{
Corresponding author: \\ Dr B Dower \\ Tel: $0215065610 / 50$ \\ Fax: 0215065619 \\ Email: brendan@capehipandknee.co.za \\ 3rd floor Life Orthopaedic Hospital \\ Vincent Pallotti Hospital \\ Alexandra Road \\ 7405 Pinelands
}

\begin{abstract}
Introduction: A rapid recovery protocol for hip and knee replacement surgery is a multidisciplinary, standardised pathway to meet the increasing demands for surgery and enhancement of recovery. This is the idea behind the recent global push by funders for cost effective, elective primary hip and knee arthroplasty. We report on a pilot study to assess the implementation and feasibility of a standardised care pathway in a South African private hospital setting.

Materials and methods: Eligible patients presenting for primary hip or knee arthroplasty were enrolled in a rapid recovery programme. The protocol that was implemented was based on current literature and international best practices. It involved members of a multidisciplinary team and the standardisation of the treatment of patients undergoing elective hip and knee arthroplasty.

Results: Forty-six patients were enrolled in the pilot study and 43 patients were successfully discharged by the third post-operative day. There were no major complications and high patient satisfaction was recorded.

Conclusion: This pilot study successfully implemented a multidisciplinary and standardised treatment protocol for hip and knee arthroplasty in a South African setting. The rapid recovery protocol proved to be safe and effective for the management of hip and knee replacements.
\end{abstract}

Key words: rapid recovery arthroplasty, short stay protocol, enhanced recovery after surgery (ERAS)

\section{Introduction}

Total joint arthroplasty has become established as a very successful method of treating end-stage arthritis of the hip and knee with excellent long-term outcomes. ${ }^{1,2}$ With an ageing population, there is an increased demand for services relating to joint arthroplasty. Individual patient's demands and expectations are also increasing, with patients desiring a quicker return to normal functioning with minimal discomfort after their joint replacement surgery.

In today's climate of limited resources and global financial strain we need to investigate ways of containing cost while enabling and maintaining safe effective treatment with optimised results. Any alteration to the surgical management of hip and knee arthroplasty should never compromise patient safety and outcomes. 
Within the analysis of the joint replacement care pathway, there are aspects of the pathway that can be optimised and standardised to reduce cost. The cost of the prosthesis and that of the surgical and anaesthetic clinical services are relatively fixed; however, effective ways to reduce the cost of the joint replacement surgery may be:

- Patient pre-operative education to set expectations regarding length of hospital stay and early mobilisation

- Pre-operative anaesthetic assessment to identify and mitigate potential risk that may lead to adverse patient outcomes

- Pre-operative optimisation of the patient's medical conditions and comorbidities.

- Standardisation of implant selection to ensure volumebased discounts

- Standardisation of the care pathway to ensure optimal use of theatre time and drug administration

- Limitation of high care admission where unnecessary

- Early mobilisation to reduce overall duration of hospital stay and post-operative complications.

This is the essence of the rapid recovery protocol. The first description of a standardised care pathway was used in colorectal surgery and has successfully been applied to other areas of general surgery. ${ }^{3}$ Specifically, the protocol has found a niche in elective orthopaedic surgery. Orthopaedic-specific standardised pathways were first developed and rolled out in high-volume specialised orthopaedic centres in the United Kingdom and have subsequently been utilised in smaller units. The use of orthopaedic-specific rapid recovery protocols has resulted in improved patient satisfaction and reduced morbidity and cost. ${ }^{4}$

Our aim was to assess the feasibility of the implementation and utilisation of an orthopaedic-specific standardised care pathway in a South African private hospital setting. We conducted a pilot study to assess the safety and efficacy of using the pathway in our practice.

\section{Materials and methods}

A pilot study was conducted at a dedicated private orthopaedic hospital and a senior author performed all the surgeries. Informed consent to participate in the pilot study was obtained from all patients.

We report an audit of our series of patients.

Exclusion criteria were:

1. American Society of Anesthesiology (ASA) score of greater than 3, or poorly controlled medical conditions

2. Patients with morbid obesity with a body mass index (BMI) greater than 40

3. Any patients identified to require intensive or high care admission post-surgery

4. Patients with cognitive impairment

5. Patients with poor home circumstances or support
The standardised care pathway described was developed by the senior authors and is based on experience, current literature and international best practices. It was developed for the South African context in consultation with a multidisciplinary team that included anaesthetic, physiotherapy and nursing staff.

This multidisciplinary approach is centred on patient education, and in developing a positive perception that the post-operative processes will run smoothly and result in an early discharge from hospital. ${ }^{5}$

The protocol involves specific standardised interventions for each patient from the enrolment date to discharge and the final independent telephonic consultation rating the patient experience. These interventions started with various appointments in the pre-operative phase. The surgeon, anaesthetist, practice nurse and physiotherapist assessed the patients prior to surgery. The principal goal was preoperative patient optimisation to ensure early mobilisation post-procedure with early discharge.

The surgical episode was standardised as far as possible in terms of prosthesis company choice, intra-operative care, and post-operative regimens. All patients returned to a standard orthopaedic ward. Patients were operated on in groups and grouped together in 2- and 4-bed wards with a senior nursing sister assigned to the ward to provide postoperative care.

The key to the process was constant reinforcement of the standardised care pathway.

\section{Rapid recovery protocol}

\section{Pre-operative phase}

The process begins at the first consultation when the decision has been made for joint replacement surgery and extends beyond hospital discharge. The surgeon introduces the concept of rapid recovery pre-operatively while the anaesthetist and physiotherapist continue to affirm the concept.

The anaesthetist assesses the patient's medical fitness for the procedure two to four weeks pre-operatively to: 1 . ensure that medical conditions and comorbidities are optimised for the surgery; 2 . identify and mitigate anaesthetic and medical risk; and 3. obtain full informed consent for the anaesthetic.

The physiotherapy team assesses the patient, and weekly sessions of pre-habilitation over a four-week period is undertaken. These visits educate and reinforce the concepts of rapid mobilisation and enhanced post-operative recovery. The patients are fitted with crutches, given exercises and taught stretching techniques pre-operatively. Written educational material is provided to the patients regarding the procedure and the recovery process. ${ }^{6}$ Where possible the physiotherapy sessions are conducted in 
small groups with patients who will have their surgery scheduled on the same day. This allows for a shared patient experience and camaraderie.

Importantly, the patient's home and support circumstances are assessed to ensure early and safe discharge post-surgery. The practice nurse makes contact with the patient and will become the patient's main contact point for any support that the patient may need post-operatively.

\section{Peri-operative care}

All patients are admitted on the morning of surgery. They are encouraged to maintain oral hydration up to 2 hours pre-operatively. All receive a premedication of $1 \mathrm{~g}$ paracetamol, $10-20 \mathrm{mg}$ temazepam and $300 \mathrm{mg}$ gabapentin. ${ }^{7-11}$

Ideally a regional spinal anaesthetic with sedation is administered. A prophylactic dose of antibiotics is administered within 30 minutes of skin incision and an antiemetic is administered to decrease postoperative nausea. ${ }^{12}$ Meticulous cautery of any bleeding points and intravenous tranexamic acid is used to decrease blood loss. ${ }^{13-15}$ Surgical drains and the use of urinary catheters are avoided. Limited intravenous fluids are given intra-operatively, sufficient only to replace blood and fluid losses. Meticulous haemostasis is maintained throughout surgery so as to limit blood loss.

A large volume of local anaesthetic, bupivacaine $0.125 \%$ with adrenalin, is administered into the joint by the surgeon. This is to decrease blood loss and to reduce post-operative pain. ${ }^{16}$ Three volumes of $50 \mathrm{ml}$ are used during the surgery. The first volume is used just after the skin incision and is injected subcutaneously. The second volume is used at the time of prosthesis implantation; in total hip replacements this is injected into the capsule and surrounding structures and in total knee replacements this is injected through the posterior capsule either side of the neurovascular bundle. The final volume is used immediately prior to deep layer closure and is injected into the soft tissues specifically targeting the incised muscle surfaces. Before leaving theatre, all patients who are able to receive a non-steroidal anti-inflammatory agent, receive a suppository to aid post-operative pain control.

Once back in the ward intravenous fluids are stopped; patients are encouraged to start oral hydration. As soon as normal muscle tone has returned, the patient is seen by physiotherapy and mobilised. ${ }^{17}$ This usually is possible six hours after the spinal anaesthetic was performed. DVT prophylaxis is provided; Clexane $40 \mathrm{mg}$ subcut daily from day 1 and then discharged on Xarelto $10 \mathrm{mg}$ daily for three weeks.
Nursing staff are actively involved in the postoperative care with routine observations and hydration as well as the regular administration of oral non-opiate analgesia. Patients are routinely given $300 \mathrm{mg}$ gabapentin twice daily (two days), $1 \mathrm{~g}$ paracetamol four times a day, $400 \mathrm{mg}$ ibuprofen three times a day. In addition, $10 \mathrm{mg}$ of oxycodone 12 hourly (three doses) is available to patients identified in the pre-operative period to have high analgesic requirements. Rescue analgesia in opiate form is prescribed but rarely required. Once mobilised, patients are encouraged to dress in comfortable clothing and to self-care as much as possible.

Patients are mobilised as often as possible, ideally at least two to three times a day with the physiotherapist until discharge. The decision as to the timing of discharge is dependent on the multi-disciplinary team being satisfied with all aspects of the patient's recovery, but is planned for the third post-operative day.

The primary outcome measured in the pilot was length of hospital stay. Secondary outcomes evaluated included the need for readmission; the use of inpatient opiate analgesia for breakthrough pain; wound or dressing complications requiring intervention after discharge; and the need for postoperative urinary catheterisation.

Lastly, all patients were contacted telephonically at three months following surgery by an independent investigator. Patient satisfaction and willingness to undergo the same standardised care pathway again were assessed. Western Ontario and McMaster Universities Osteoarthritis Index $\left(\mathrm{WOMAC}^{18}\right.$ ) scores were also measured at 3 months postoperatively by a physiotherapist and were compared to scores obtained during the pre-operative physiotherapy assessment.

\section{Results}

Forty-six patients undergoing hip and knee joint replacement surgery were enrolled. Twelve males and 33 females were included. The mean age was 60.4 years (range 25-89). Thirty-two underwent total hip replacements and 14 had total knee replacements.

Forty-three of $46(93 \%)$ patients were discharged by the third post-operative day. The average length of hospital stay was 2.8 days with a range of 2 to 4 days. This is well below the National Joint Registry of England and Wales average of 6.3 days. ${ }^{2}$ Factors preventing discharge within 3 days (target) were post-operative pain and orthostatic hypotension.

Three elderly female patients required catheterisation for urinary incontinence on the first night following surgery. Eight patients required additional IMI opiate analgesia during their inpatient stay. Seven patients required an additional change of dressing at home within 
the first post-surgical week. One total knee replacement required a manipulation at six weeks for poor range of motion. No major complications, including DVT or PE were recorded within 90 days of surgery. There were no other readmissions or re-operations.

All patients reported that they were extremely satisfied with the surgical result at three months; however, one patient said that they would prefer not to undergo a 'rapid recovery process' again, as they felt hurried and stressed by the early discharge.

The mean pre-operative WOMAC score was 35, compared to a mean score of 85 at three months post-procedure.

\section{Discussion}

Our experience suggests that a rapid recovery protocol with an enhanced discharge pathway is safe, feasible and acceptable in a South African private hospital setting. This protocol requires multidisciplinary team contributions and active involvement by the patients. The findings demonstrate that one can reduce the length of hospital stay and that high care admissions are not routinely required post joint replacement surgery. Previously our average length of stay was 5.5 days with one day in high care. This has resulted in an immediate cost saving of R10 000. We have subsequently implemented aspects of this protocol for suitable total arthroplasty patients and are hoping to extend this practice to a parallel service in a state hospital.

Patient pre-operative assessments, optimisation and education, in conjunction with protocol-driven perioperative care and safe early mobilisation has resulted in decreased length of hospital stay and no post-operative high care. This has financial ramifications for both public and private institutions as well as health care funders and government. There are also other potential health benefits that have not been explored in detail in this pilot study. The risk of deep vein thrombosis could be reduced as a result of the rapid mobilisation process. Shorter hospital stays may also result in fewer hospital-acquired infections. The usage of tranexamic acid with meticulous cautery, and minimising the usage of site drains and urine catheterisation, reduces the risk of potential prosthesis-related infection and catheter-related urinary tract infections.

The WOMAC score improvements seen in our study population are comparable to those in the published literature. ${ }^{19}$

An additional benefit has been the improved inter-disciplinary communications as a result of our close working together in the care of our patients. We have noticed improved staff morale when dealing with the rapid recovery patients as all team members feel they have an important role to play in the recovery of these patients.

The primary aim of assessing the implementation, feasibility and acceptability of a standardised enhanced discharge pathway in the specific setting of the South African private sector has been achieved by this pilot study. Considerable additional information regarding length of hospital stay, level of care and analgesia required has been gathered. This study is an internal pilot study and it may form part of a larger study assessing the outcomes of implementing a standardised pathway in the wider South Africa context. It is purely descriptive and the weakness of this study is the lack of comparative controls. A longer-term prospective randomised trial comparing a rapid recovery group with a traditional care cohort would be an ideal study design; we hope to publish a larger series in future.

In conclusion, a standardised enhanced discharge pathway with a rapid mobilisation protocol in a South African private hospital setting is a safe and effective way of managing total joint arthroplasty patients without compromising surgical care or rehabilitation.

\section{Compliance with ethics guidelines}

This article is the sole work of the authors and no financial benefit has been or will be received, related to this work.

Informed consent to participate in the pilot study was obtained from all patients; institutional ethics approval was not obtained.

Dr Dower and Dr Springfield are directors of ICPS.

\section{References}

1. Learmonth ID, Young C, Rorabeck C. The operation of the century: total hip replacement. Lancet 2007 Oct 27;370(9597):1508-19.

2. No authors listed. National Joint Registry for England and Wales 10th Annual Report, 2013. www.njrcentre.org.uk

3. Kehlet H. Multimodal approach to control postoperative pathophysiology and rehabilitation. Br J Anaesth. 1997 May;78(5):606-17.

4. Larsen K, Hansen TB, Thomsen PB, Christiansen T, Soballe K. Cost-effectiveness of accelerated perioperative care and rehabilitation after total hip and knee arthroplasty. J Bone Joint Surg Am. 2009 Apr;91(4):761-72.

5. Wainwright T, Middleton R. An orthopaedic enhanced recovery pathway, Current Anaesthesia \& Critical Care (2010), doi10.1016/j.cacc.2010.01.003

6. Yoon RS, Nellans KW, Geller JA, Kim AD, Jacobs MR, Macaulay W. Patient education before hip or knee arthroplasty lowers length of stay. J Arthroplasty 2010 Jun;25(4):547-51.

7. Mallory TH, Lombardi AV, Fada RA, Dodds KL, Adams JB. Pain management for joint arthroplasty: preemptive analgesia. J Arthroplasty 2002 Jun 17;4 (Suppl 1):129-33.

8. Bromley L. Pre-emptive analgesia and protective medication. What is the difference? Biomed Pharmacotherapy. 2006 Aug;60(7):336-40.

9. Buvanendran A, Kroin JS, Della Valle CJ, Kari M, Moric M, Tuman KJ. Perioperative oral pregabalin reduces chronic pain after total knee arthroplasty: a prospective, randomized, controlled trial. AnaesthEAnalg 2010 Jan 1;110(1):199-207. 
10. Carmichael NM, Katz J, Clarke H, Kennedy D, Kreder HJ, Gollish J, Mcartney CJ. An intensive perioperative regimen of pregabalin and celecoxib reduces pain and improves physical function scores six weeks after total hip arthroplasty: a prospective randomized controlled trial. Pain Res Manag. 2013 May-Jun;18(3):127-32.

11. White PF, Kehlet H, Neal JM, Schricker T, Carr DB, Carli F. The role of the anesthesiologist in fast-track surgery: from multimodal analgesia to perioperative medical care. AnesthAnalg 2007 Jun;104(6):1380-96.

12. Backes JR, Bentley JC, Politi JR, Chambers BT. Dexamethasone reduces length of hospitalization and improves postoperative pain and nausea after total joint arthroplasty: A prospective, randomized controlled trial. J Arthroplasty 2013 Sep;28(8 suppl):11-17.

13. Sukeik M, Alshryda S, Haddad FS, Mason JM. Systemic review and meta-analysis of the use of tranexamic acid in total hip replacement. J Bone Joint Surg Br. 2011 Jan;93(1):39-46.

14. Alshryda P, Sarda P, Sukeik M, Nargol A, Blenkinsopp J, Mason JM. Tranexamic acid in total knee replacement: a systematic review and meta-analysis. J Bone Joint Surg Br. 2011 Dec;93(12):1577-85.

15. Irisson E, Hemon Y, Pauly V, Parratte S, Argenson JN, Kerbaul F. Tranexamic acid reduces blood loss and financial cost in primary total hip and knee replacement surgery. Orthop and Traumatol Surg Res 2012 Sep;98(5):477-83.
16. Busch CA, Whitehouse MR, Shore BJ et al. The efficacy of periarticular multimodal drug infiltration in total hip arthroplasty. ClinOrthopRelat Res 2010 Aug;468(8):215259.

17. Juliano K, Edwards D, Spinello D, Capizzano Y, Epelman E, Kalowitz J, Lempel A, Ghomrawi H. Initiating physical therapy on the day of surgery decreases length of stay without compromising functional outcomes following total hip arthroplasty. HSS J 2011 Feb;7(1):1620.

18. Bellamy N, Buchanan WW, Goldsmith $\mathrm{CH}$, Campbell J, Stitt LW. Validation study of WOMAC: a health status instrument for measuring clinically important patient relevant outcomes to antirheumatic drug therapy in patients with osteoarthritis of the hip or knee. J Rheumatology 1988 Dec;15(12):1833-40.

19. Amlie E, Havelin LI, Furnes O, Baste V, Nordsletten L, Hovik O, Dimmen S. Worse patient-reported outcome after lateral approach than after anterior and posterolateral approach in primary hip arthroplasty. ActaOrthop 2014 Jun23;1-7. (Epup ahead of print)

This article is also available online on the SAOA website (www.saoa.org.za) and the SciELO website (www.scielo.org.za). Follow the directions on the Contents page of this journal to access it. 